\title{
Article
}

\section{Machiavellianism, emotional manipulation, and friendship functions in women's friendships}

\author{
Abell, Loren, Brewer, Gayle, Qualter, Pamela and Austin, Elizabeth \\ Available at http://clok.uclan.ac.uk/12508/ \\ Abell, Loren, Brewer, Gayle ORCID: 0000-0003-0690-4548, Qualter, Pamela \\ and Austin, Elizabeth (2016) Machiavellianism, emotional manipulation, and \\ friendship functions in women's friendships. Personality and Individual \\ Differences, 88 . pp. 108-113. ISSN 01918869
}

It is advisable to refer to the publisher's version if you intend to cite from the work. http://dx.doi.org/10.1016/j.paid.2015.09.001

For more information about UCLan's research in this area go to http://www.uclan.ac.uk/researchgroups/ and search for <name of research Group>.

For information about Research generally at UCLan please go to http://www.uclan.ac.uk/research/

All outputs in CLoK are protected by Intellectual Property Rights law, including Copyright law. Copyright, IPR and Moral Rights for the works on this site are retained by the individual authors and/or other copyright owners. Terms and conditions for use of this material are defined in the policies page.

\section{CLoK}

Central Lancashire online Knowledge www.clok.uclan.ac.uk

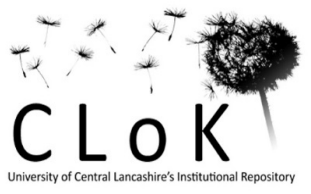




\section{Machiavellianism, emotional manipulation, and friendship functions in women's friendships}

Machiavellianism and emotional manipulation has not been investigated in friendships. The current studies investigated Machiavellianism, emotional manipulation, and six friendship functions in women's same-sex friendships. For study 1 , women $(N=221)$ completed the Mach IV, emotional manipulation measure (with reference to their own behaviour and their friend's behaviour), mood worsening and use of inauthentic displays from the managing emotions of others scale, and the friendship functions measure. Machiavellianism predicted the self-perceived ability to employ emotional manipulation towards a same-sex friend and perceiving their friend to use emotional manipulation towards them. Machiavellianism predicted lower scores on all six friendship functions. For study 2, women $(N=186)$ completed the Mach IV, the modified emotional manipulation measure (with reference to their own behaviour and their friend's behaviour), and the friendship functions measure. Women high on Machiavellianism reported using emotional manipulation more frequently towards their same-sex friend and perceived their same-sex friend to frequently use emotional manipulation towards them. Machiavellianism predicted lower scores on five of the friendship functions. These studies demonstrated that women higher on Machiavellianism employ emotional manipulation in their same-sex friendships. Women with higher Machiavellianism scores also perceived that they themselves were manipulated by their friend.

Keywords: Machiavellianism; emotional manipulation; friendship.

\section{Introduction}

Men and women with high levels of Machiavellianism, characterised by emotional detachment, cynicism, and a manipulative interpersonal style (Christie \& Geis, 1970), seek closeness from others in order to manipulate and exploit (Ináncsi, Láng, \& Bereczkei, 2015). These individuals are low on empathy, not connected to their own or other peoples' emotions, and hold negative representations of others (Black, Woodworth, \& Porter, 2013; Ináncsi et al., 2015; Wai \& Tiliopoulos, 2012, Wastell \& Booth, 2003), which may facilitate their use of manipulation. Machiavellianism 
influences a variety of adult relationships, and, given friendship is the most common form of social relationship (Blieszer \& Adams, 1992), it is important to investigate Machiavellianism in this context. Men and women with high levels of Machiavellianism do engage in friendships, but report low friendship quality (Abell, Lyons \& Brewer, 2014; Aitken \& Lyons, 2010). This is unsurprising given the high levels of suspicion, cynicism, and emotional detachment associated with Machiavellianism. Research also demonstrates that adults with high Machiavellianism levels select opposite-sex friends who are kind (Jonason \&Schmitt, 2012). This may indicate a preference for friends that can be easily exploited. Furthermore, Machiavellianism is associated with the self-reported manipulation of an opposite and same-sex friend through strategies such as the use of 'silent-treatment' and coercion (Jonason \& Webster, 2012).

Women's friendships, in particular, may provide opportunities to exploit and manipulate. Women report a greater focus on interpersonal relationships (Su, Rounds, \& Armstrong, 2009), which may in part reflect a greater reliance on female friends when faced with adaptive problems such as finding a mate (Jonason \& Schmitt, 2012; Silverman \& Choi, 2005). Women spend more time discussing feelings and personal information and their friendships tend to be dyadic in nature, which does not allow for substitute partners if relationships break down (Benenson \& Christakos, 2003; David-Barrett et al., 2015; Vigil, 2007). This focus on exclusive friendships characterised by information sharing may provide a context for specific types of manipulation to take place.

Women tend to use relational aggression as a manipulation strategy and, overall, women's manipulation is reported to require more subtle methods (Wilson, Near, \& Miller, 1996). This may be related to the risks of engaging in physical aggression (Campbell, 1999), but, also, it may be seen as a socially acceptable way for women to relate to each other and to build relationships (Miller-Ott \& Kelly, 2013). Relational aggression refers to behaviour that harms others through the manipulation of relationships using exclusion, gossip, and rumours (Archer \& Coyne, 2005; Xie, Cairns \& Cairns, 2005). Relational aggression demands support from peers and/or friends because it requires them to listen to the gossip, help spread rumours, exclude the target individual(s), whilst also offering their own thoughts about the target (Miller-Ott \& Kelley, 2013). Therefore, it involves trust from others to participate and trust that they will not betray them to the target. 
Although relational aggression is more subtle than direct aggression, it may be a problematic strategy for women with high levels of Machiavellianism to engage in. The use of relational aggression requires a level of trust and connection to others, and requires involvement from peers/friends. Machiavellianism is, however, characterised by distrust, suspicion, and cynicism (Christie \& Geis, 1970), making relational aggression incompatible with Machiavellianism. The greater number of individuals that engage in relational aggression may also increase the likelihood of getting caught, which individuals (particularly those with high levels of Machiavellianism) wish to avoid. Although Machiavellianism is related to women's use of relational aggression towards friends online (Abell \& Brewer, 2014), this may reflect the absence of face-to face contact and the decreased reliance on others when engaging in relational aggression in this context.

It may be more beneficial to employ subtle manipulation tactics towards a close friend rather than relying on others to help employ manipulation tactics. One such tactic is emotional manipulation, which includes the use of strategies to manage the emotions of others (Austin, Farrelly, Black, \& Moore, 2007; Austin \& O'Donnell, 2013). Machiavellianism is associated with the use of emotional manipulation (Austin et al., 2007) and includes such tactics as strategically paying the other person a compliment and reassuring others so they will go along with what the individual wants. However, the use of emotion manipulation by people high on Machiavellianism has not been investigated in the context of friendship. Furthermore, Machiavellianism is associated with two particular strategies of emotional manipulation that are used when managing other people's emotions; worsening strategies (e.g., undermining another person's confidence, using criticism) and inauthentic strategies (e.g., eliciting sympathy, sulking to get own way). Emotional manipulation (including the use of emotion managing strategies of mood worsening and inauthentic strategies) only requires one target individual and the perpetrator, rather than the trust and connection of others that are needed during relational aggression; it is also covert, reducing the chance of detection both by the target and others. The use of emotional manipulation may reduce the likelihood of relationship breakdown, reputational damage, and the challenge of then finding a new same-sex friend.

In addition to Machiavellian women's self-reported ability to use emotional manipulation, there may also be a relationship between Machiavellianism and women's perception that their friend 
uses emotional manipulation directed towards them. For example, Machiavellianism is associated with viewing others as weak (e.g., Black et al., 2013), therefore, women with higher levels of Machiavellianism may view others (in particular their same-sex friend) as incapable of employing manipulation towards them. Machiavellianism is, however, also associated with distrust of others and the belief that people will try to exploit them (Christie \& Geis, 1970). This may indicate that women with higher Machiavellianism scores will perceive their friend as trying to exploit them by employing emotional manipulation.

Previous research suggests that emotional manipulation is likely to be deployed by women with higher Machiavellianism scores in their close friendships with other women. These women may also report that they are targeted in this way by their close female friends. The relationship between emotional manipulation and Machiavellianism in friendship has not previously been investigated. Specifically, we report results from two studies which investigate women's perceived ability to manipulate a close same-sex friend and the perception that they themselves are manipulated (study 1) and women's self-reported frequency of employing emotional manipulation and their perception of the frequency that emotional manipulation is used towards them (study 2).

\section{Study 1}

Study 1 investigates whether Machiavellianism is associated with the use of emotional manipulation in friendship and the use of two specific emotional manipulation tactics (worsening and inauthentic strategies). Based on previous research (Austin et al., 2007; Austin \& O’Donnell, 2013) and the potential benefits of using emotional manipulation (e.g., less reliance on others, reduced chance of getting caught), we predict that higher levels of Machiavellianism will be associated with the use of emotional manipulation (including the use of inauthentic and mood worsening strategies) towards a close female friend. In addition, this study explores the relationship between Machiavellianism and the perception of manipulation. Previous research has shown that Machiavellianism is related to poor friendship quality (Abell et al., 2014; Aitken \& Lyons, 2010), but has not explored how individuals with higher levels of Machiavellianism view the functions of friendship. Therefore, the relationship between Machiavellianism and six functions of friendship will be considered. The six functions are companionship, help, intimacy, reliable alliance, self-validation, 
and emotional security. Although friendships may offer a number of advantages for individuals with higher Machiavellianism scores, such as help in achieving their own goals, we suggest that Machiavellianism will predict lower scores on all six friendship functions. The emotional detachment and cynicism that characterises Machiavellianism may result in women with higher scores reporting low levels of these functions because of the broad negative view they have of others.

\section{Method}

\subsection{Participants}

Participants were 221 women aged 18 to $69\left(M_{\text {age }}=27.55, S D=11.17\right)$ with an average friendship length of 123.58 months $(S D=92.67)$. The participants were a volunteer sample from online research websites and social networking sites and received no financial reward for participation.

\subsection{Measures}

\section{Machiavellianism}

Machiavellianism was assessed with the 20-item Mach-IV scale (Christie \& Geis, 1970), which measures morality, cynicism, and manipulative interpersonal style. Example items from the scale include "The best way to handle people is to tell them what they want to hear" and "It is wise to flatter important people”. Participants responded on a 7-point Likert scale $(1=$ strongly disagree; $7=$ strongly agree). Ten items were reverse scored, such that higher scores represent higher Machiavellianism, with total scores used in the analysis. The scale demonstrated good reliability $\alpha=$ .73.

\section{Emotional Manipulation}

Emotional manipulation was measured with the 10-item Emotional Manipulation measure (Austin et al., 2007) that describes general emotional manipulation strategies. Items include "I know how to embarrass someone to stop them behaving in a particular way" and "I can use my emotional skills to make others feel guilty". Participants responded on a five-point Likert scale ( $1=$ strongly disagree; $5=$ strongly agree . In this study the statements were altered slightly to reflect emotional manipulation specifically towards a friend. For example "I know how to embarrass my friend to stop them behaving in a particular way". Items were them summed to generate an emotional manipulation 
score. The scale demonstrated excellent reliability $\alpha=.87$. Participants then completed the scale for a second time with reference to their friend's manipulative behaviour towards them. For example " $M y$ friend knows how to embarrass me to stop me behaving in a particular way". The scale demonstrated excellent reliability $\alpha=.88$.

Managing Emotions of Others

Two subscales, mood worsening and use of inauthentic displays for self-serving purposes, from the Managing Emotions of Others Scale (Austin \& O’Donnell, 2013) were used in this study. Items were summed to create two subscale totals. The original mood worsening subscale consisted of 13 statements that include the use of criticism and undermining confidence. In this study, four items were removed which were part of the Emotional Manipulation Scale (as mood worsening and emotional manipulation both involve managing others emotions). Items in the scale include " $I$ sometimes try to undermine another person's confidence" and "I use displays of anger to motivate others". The scale demonstrated excellent reliability $\alpha=.87$.

The original inauthentic moods subscale included 11 statements. They include statements that assess the use of flattery and inducing jealousy. One item was removed from the scale because it formed part of the Emotional Manipulation Scale. Emotional manipulation and using inauthentic moods both incorporate managing another person's emotions. Items include “I sometimes sulk to get someone to change their behavior" and "If I want someone to do something for me, I am especially nice to them before asking". Participants responded on a five-point scale for both subscales $(1=$ strongly disagree; 5 = strongly agree). In this study statements were altered to specifically reflect behaviour with a friend, for example "I sometimes sulk to get my friend to change their behavior". The scale demonstrated excellent reliability $\alpha=.88$.

\section{Friendship Functions}

Friendship functions were measured with the McGill Friendship Functions short-form questionnaire (MFQ-FF; Mendelson \& Aboud, 1999). This is a 30-item measure that assesses six functions of friendship: stimulating companionship; help; intimacy; reliable alliance; self-validation; and emotional security. Totals were calculated for each subscale. Participants were asked to imagine that each statement referred to their close friend. Stimulating companionship refers to spending time 
with their friend that results in feelings of enjoyment e.g., “ is fun to sit and talk with". Help refers to providing assistance and advice to meet the individual's needs and goals e.g., "__ helps me when I need it". Intimacy refers to providing an environment where personal thoughts and feelings can be expressed safely .e.g., "__ is easy to talk to about private things". Reliable alliance refers to counting on the continuing loyalty of their friend: e.g., " would stay my friend even if we argued". Selfvalidation refers to their friend as being encouraging and reassuring and helping to validate ones selfworth e.g., “__ makes me feel special”. Emotional security refers to the provision of comfort provided by the friend in novel and/or frightening situations e.g., "___would make me feel better if I were worried". Participants respond on a 9-point scale $(0=$ never; $8=$ always $)$. The subscales demonstrated excellent reliability ranging from $\alpha=.89$ to $\alpha=.92$.

\subsection{Results}

\subsection{Correlations}

Correlations with means for the measures are shown in Table 1. Machiavellianism significantly positively correlated with emotional manipulation, mood worsening strategies, inauthentic strategies, and perceiving emotional manipulation from a friend. Machiavellianism also demonstrated significant negative correlations with all six friendship functions subscales (companionship, help, intimacy, reliable alliance, self-validation, and emotional security).

\subsection{Machiavellianism and Manipulation}

Robust regressions were conducted with bootstrapping set at 1000 samples, with a $95 \%$ bias corrected accelerated confidence interval. Four separate regressions were conducted to explore the relationship between Machiavellianism and manipulation in same-sex female friendship. The four models were significant in predicting perceived ability to employ emotional manipulation towards a same-sex female friend $(F(3,165)=11.54, p<.001)$, the use of mood worsening tactics $(F(3,163)=$ $15.35, p<.001)$, the use of inauthentic strategies $(F(3,162)=12.46, p<.001)$, and perceiving emotional manipulation from a friend $(F(3,154)=6.53, p<.001)$. Machiavellianism (after controlling for age and friendship length at Step 1), individually predicted the use of emotional manipulation $(\beta=.37, t=5.14, p=.001)$, the use of mood worsening tactics $(\beta=.41, t=5.81, p=$ $.001)$, and the use of inauthentic strategies towards a same-sex friend $(\beta=.32, t=4.40, p=.001)$. 
These findings suggest that higher levels of Machiavellianism increased self-reported ability to use emotional manipulation, mood worsening strategies, and inauthentic strategies directed at a close female friend. Furthermore, the model for Machiavellianism predicted perceiving emotional manipulation from a friend $(\beta=.24, t=3.16, p=.005)$, suggesting that women with higher Machiavellianism scores perceived their same-sex friend to have the ability to use emotional manipulation towards them.

\subsection{Machiavellianism and Friendship Functions}

Separate regressions (with bootstrapping) were conducted to explore the relationship between Machiavellianism and six functions of friendship. The six models were significant in predicting companionship $(F(3,151)=9.02, p<.001)$, help $(F(6,175)=10.42, p<.001)$, intimacy $(F(3,151)$ $=5.90, p<.001)$, reliable alliance $(F(3,151)=7.07, p<.001)$, self-validation $(F(3,151)=9.28, p<$ $.001)$, and emotional security $(F(3,151)=4.80, p=.003)$. Machiavellianism (after controlling for age and friendship length at Step 1), individually predicted perceiving their friend to provide less companionship $(\beta=-.35, t=-4.57, p=.001)$, less help $(\beta=-.40, t=-5.30, p=.001)$, less intimacy $(\beta$ $=-.33, t=-4.20, p=.001)$, to be less of a reliable ally $(\beta=-.34, t=-4.34, p=.003)$, to provide less self-validation $(\beta=-.40, t=-5.26, p=.001)$, and less emotional security $(\beta=-.30, t=-3.77, p=.001)$.

\section{Study 1 Discussion}

Study 1 demonstrated that women with higher levels of Machiavellianism report the ability to employ emotional manipulation directed at a close same-sex friend. This included tactics such as making their friend feel ashamed, embarrassed, and/or guilty. Furthermore, Machiavellianism was also associated with women's use of mood worsening tactics such as using anger and knowledge of their friend's emotional triggers to manipulate them, and the use of inauthentic strategies such as sulking and deliberately making their friend feel jealous. Women with higher Machiavellianism scores also perceived their friend to be emotionally manipulative towards them. This may stem from viewing others as distrustful, controlling, and demanding, and showing sensitivity to unfair treatment (Christie \& Geis, 1970; Schmitt, Gollwitzer, Maes, \& Arbach, 2005; Sherry, Hewitt, Besser, Flett, \& Klein, 2006).

Previous research has demonstrated that Machiavellianism is associated with poor friendship 
quality (Abell et al., 2014; Lyons \& Aitken, 2010). This study further explored the influence of Machiavellianism in the context of friendship, with the inclusion of subscales measuring separate friendship functions. Machiavellianism was associated with lower scores on all of the six friendshipfunctions subscales. Although same-sex friendships are often labelled as being highly important to women and provide a variety of functions and resources (Jonason \& Schmitt, 2012; Silverman \& Choi, 2005; Vigil, 2007), women with higher levels of Machiavellianism may view such functions as unnecessary. It is not surprising that women with higher levels of Machiavellianism have reported that their friend provides them with less companionship, help, intimacy, reliable alliance, self-validation, and emotional security. These six functions require a degree of emotional attachment and trust, whereas Machiavellianism is associated with distrust, suspicion, and independence.

We now investigate Machiavellianism and emotional manipulation in women's friendships from a different angle, by using the modified emotional manipulation measure (Hyde \& Grieve, 2014) which measures the frequency with which individuals use emotionally manipulation. This scale is a modified version of Austin et al.’s (2007) emotional manipulation scale. Hyde and Grieve (2014) conducted a factor analysis which revealed a distinction between perceived ability to emotionally manipulate (Austin et al., 2007) and willingness to emotionally manipulate (Hyde \& Grieve, 2014). Results may indicate that not only do women with higher Machiavellianism scores report an ability to use emotional manipulation towards a close same-sex friend but also report more frequent use of emotional manipulation towards a close same-sex friend.

\section{Study 2}

Study 1 investigated whether Machiavellianism was associated with the perceived ability to emotionally manipulate a same-sex female friend. Study 2 now investigates whether Machiavellianism is associated with emotional manipulation frequency in women's friendship. We predict that higher levels of Machiavellianism will be associated with the greater self-reported use of emotional manipulation towards a close female friend. In addition, this study explores the relationship between Machiavellianism and the perception of emotional manipulation frequency from their friend.

\section{Method}




\subsection{Participants}

Participants were 186 women aged 18 to $66\left(M_{\text {age }}=23.65, S D=8.34\right)$ with an average friendship length of 112.59 months $(S D=84.36)$. The participants were a volunteer sample from online research websites and social networking sites and received no financial reward for participation.

\subsection{Measures}

Study 2 also employed the Mach IV ( $\alpha=.69)$ and the Friendship Functions short-form questionnaire (reliabilities ranged from: $\alpha=.86$ to: $\alpha=.90$ ) used in study 1 . In addition, the modified Emotional Manipulation Measure (Hyde \& Grieve, 2014) was used. This scale is a modified version of Austin et al.'s (2007) Emotional Manipulation Scale and measures the frequency of emotional manipulation. Hyde and Grieve (2014) conducted a factor analysis, which revealed a distinction between perceived ability to emotionally manipulate (Austin et al., 2007) as measured in study 1 and willingness to emotionally manipulate (Hyde \& Grieve, 2014) which is being investigated in study 2. Questionnaire items include "How often do you use your emotional skills to make your friend feel guilty" and "How often do you embarrass your friend to stop them behaving in a particular way". Participants responded on a 5 -point scale $(1=$ Never to $5=$ Daily $)$ and items were summed to create a total score. As in study 1, participants completed this measure twice: first with reference to their own behaviour towards a close same-sex female friend $(\alpha=.81)$; and, second, with reference to their close same-sex friend's behaviour $(\alpha=.86)$.

\subsection{Results}

\subsection{Correlations}

Correlations with means are shown in Table 2. Machiavellianism demonstrated significant positive correlations with willingness to engage in emotional manipulation and perceived willingness of the friend to employ emotional manipulation, and significantly negatively correlated with intimacy, reliable alliance, and emotional security friendship functions.

\subsection{Machiavellianism and Emotional Manipulation Frequency}

Two separate regressions were conducted (with bootstrapping) to explore the relationship 
between Machiavellianism and frequency of emotional manipulation towards a close same-sex female friend. Both models significantly predicted emotional manipulation frequency towards a same-sex female friend $(F(3,166)=10.93, p<.001)$ and perception that their friend frequently used emotional manipulation towards them $(F(3,166)=7.35, p<.001)$. Machiavellianism (after controlling for age and friendship length at Step 1), individually predicted using emotional manipulation more frequently towards a friend $(\beta=.32, t=4.49, p=.001)$. Furthermore, Machiavellianism was associated with the perception that their friend used emotional manipulation more frequently towards them $(\beta=.20, t=$ $2.67, p=.007)$.

\subsection{Machiavellianism and Friendship Functions}

The model for companionship approached significance $(F(3,166)=2.61, p=.054)$ and the five remaining models were significant in predicting help $(F(3,166)=5.29, p=.002$, intimacy $(F(3$, $166)=4.38, p=.005)$, reliable alliance $(F(3,166)=5.89, p=.001)$, self-validation $(F(3,166)=$ $3.41, p=.019)$, and emotional security $(\mathrm{F}(3,166)=5.08, p=.002)$. As in study 1 , after controlling for age and friendship length, Machiavellianism significantly predicted viewing their friend to provide less companionship $(\beta=-.15, t=-1.98, p=.033)$, less help $(\beta=-.18, t=-2.40, p=.022)$, less intimacy $(\beta=-.23, t=-3.03, p=.005)$, and to provide less emotional security $(\beta=-.24, t=-3.20$, $p=.002)$. Machiavellianism and perceiving their friend to be a reliable ally approached significance $(\beta=-.15, t=-1.99, p=.055)$ suggesting women with higher levels of Machiavellianism viewed their friend as being less of a reliable ally. However, no relationship was revealed for Machiavellianism and perceiving their friend to provide self-validation $(\beta=-.14, t=-1.85, p=.074)$.

\subsection{Study 2 Discussion}

Study 2 demonstrated that women with higher levels of Machiavellianism employ emotional manipulation towards a close same-sex friend more frequently than women with lower levels of Machiavellianism. This may be a tactic that is preferential when manipulating someone who is familiar. Although women with high level of Machiavellianism may not feel close or attached to their friend, the appearance of a friendship may provide an ideal context in which to use emotional manipulation. Emotional manipulation is covert, allowing these women to feel more comfortable with this strategy because there is a reduced chance of detection. Women with high levels of 
Machiavellianism may use emotional manipulation tactics to a greater degree when it becomes apparent they can use these tactics without being detected, supporting the argument that Machiavellianism is based more on environmental than biological experience and that it may be a learnt behaviour (e.g., Veselka, Aitken, Schermier, \& Vernon, 2011). Although not investigated here, these tactics may also be successful in helping them to achieve their goals; therefore, future research should explore the success of emotional manipulation tactics and the likelihood of detection. Additionally, women with higher scores on Machiavellianism perceived their friend as frequently directing emotional manipulation towards them. As discussed earlier, the suspicion and distrust of others, viewing others as controlling and demanding, as well as demonstrating sensitivity to unfair treatment (Christie \& Geis, 1970; Schmitt et al., 2005; Sherry et al., 2006), may influence the belief that their same-sex friend is not only using emotional manipulation towards them, but is frequently using such tactics.

Supporting the results from study 1, higher levels of Machiavellianism in women were associated with viewing their friend as providing little companionship, help, intimacy, not being seen as a reliable ally, and providing little emotional security. In contrast to study 1, no relationship was found for Machiavellianism and self-validation. This finding suggests that women with higher Machiavellianism scores perceive their friend as not providing more or less encouragement and reassurance. It would be expected given the cynicism and suspicion that characterises Machiavellianism that a negative relationship would be revealed between all of the friendship functions or, given the high emotional detachment of Machiavellian individuals no relationship between Machiavellianism and all the functions would be revealed. Therefore, this finding for Machiavellianism and self-validation is unexpected and could be specific to this particular sample. Future research should explore Machiavellianism and friendship functions further.

\subsection{General Discussion}

The current studies investigated the influence of Machiavellianism on women's reported use of emotional manipulation directed at a close same-sex friend, and the perception that the participants themselves, were a target of emotional manipulation. In addition, the studies investigated the influence of Machiavellianism on six friendship functions. Previous research has established that 
Machiavellianism is associated with emotional manipulation and managing the emotions of others in general (Austin et al., 2007; Austin \& O'Donnell, 2013), but the current studies extended that research by focusing on the use of these strategies in a specific context of women's same-sex friendships.

Findings indicate that women with higher Machiavellianism scores report the ability to use emotional manipulation, mood worsening, and inauthentic strategies directed towards a close samesex friend. Furthermore, women use emotional manipulation frequently towards their close same-sex friend. The use of emotional manipulation by women may be facilitated by women's greater interest in social interaction and the expression of personal feelings in friendship. Women with higher levels of Machiavellianism may exploit this norm of female friendship by seeking interactions and closeness in order to manipulate. The characteristics of women's friendships, coupled with high Machiavellianism in one party may support the use of emotional manipulation strategies (Ináncsi et al., 2015; Su et al., 2009; Vigil, 2007). Employing these strategies towards one person may be less risky for women higher on Machiavellianism than engaging in relational aggression, which requires the assistance of others. The lack of connection to their own and their friend's emotions (Wastell \& Booth, 2003) may facilitate the use of manipulation because they do not reflect on the negative consequences for their friend. The greater frequency of this tactic may stem from learning that their friend does not confront them when using this tactic, so believe these strategies are undetected and (potentially) successful.

Women with higher Machiavellianism scores also reported that their close same-sex friend frequently engaged in emotional manipulation. Machiavellianism is associated with an overall general negative representation of others, believing other people cannot be trusted and will exploit them (Christie \& Geis, 1970; Ináncsi et al., 2015). Therefore, viewing their friend as also using emotional manipulation provides evidence that they see others, including a same-sex friend, as manipulative and trying to exploit them for their own gain. However, this study only considered the perception of emotional manipulation and did not examine whether their friend actually reported or employed emotional manipulation. Future research should measure both the perception and use of emotional manipulation as reported by both members of the friendship dyad.

In study 1 and study 2, Machiavellianism was associated with perceiving less companionship, 
help, intimacy, reliable alliance, and emotional security in their friendship. Those findings are consistent with previous research that demonstrated Machiavellian adults report low friendship quality (Abell, Lyons \& Brewer, 2014; Aitken \& Lyons, 2010). This is unsurprising given Machiavellianism is associated with emotional detachment and only seeking closeness in order to exploit another individual. They value independence and do not trust others (Christie \& Geis, 1970; Ináncsi et al., 2015). Despite women's focus on social relationships, empathy, and support in friendships (BaronCohen \& Wheelwright 2003; Su et al., 2009), having high levels of Machiavellianism reduces the need to feel emotionally close to another individual. Women with higher Machiavellianism scores may be skilled at appearing to provide this warm, close friendship context in order to maintain their relationship with their friend to ensure continual manipulation opportunities. However, one inconsistency was revealed with the relationship between Machiavellianism and self-validation. Selfvalidation refers to perceiving their friend to provide encouragement and to validate oneself as a worthwhile individual (Mendelson \& Aboud, 1999). This finding could be sample specific but requires more research to investigate Machiavellianism and how Machiavellian adults view their friendships.

It is important to note that studies 1 and 2 obtained data from one member of the friendship dyad only. In order to develop a greater understanding of Machiavellianism in women's friendship dynamics both members of the dyad should be considered. This may include investigating each friend's Machiavellianism scores and the use of emotional manipulation, the success of this strategy, and the perception (i.e., detection) of this. Research using a dyadic approach could also consider the advantages and benefits of friendships from women with higher Machiavellianism scores. This would be beneficial in understanding whether women with higher scores do view their friends as exploitation opportunities or whether there are additional advantages to the friendship. Furthermore, future research could also consider including measures of Narcissism and Psychopathy to investigate how these constructs also relate to emotional manipulation and friendship functions. The present study is also limited by the use of self-report measures and participants' willingness to disclose socially undesirable behaviour (e.g., Grovle, et al., 2012; Holden, Wheeler, \& Marjanovic, 2012), although research has demonstrated individuals disclose more undesirable behaviour in online studies (Booth- 
Kewley, Larson, \& Miyoshi, 2007). In addition, it should be noted that there is common variance in each of the two studies as participants completed the emotional manipulation measure and the modified measure twice (first based on their own behaviour then perception of their friend's behaviour). To reduce this common variance, future research should focus on directly observed manipulation by incorporating the use of observational methodology. This would allow for the detection of even more subtle behaviour and manipulation techniques that Machiavellian individuals may employ.

To conclude, the present studies investigated Machiavellianism and emotional manipulation in women's friendships, including the vulnerability to a friend's emotional manipulation. Women higher on Machiavellianism reported the ability to use emotional manipulation, reported to employ this strategy with greater frequency towards a close-same sex friend, and perceived their friend to frequently employ emotional manipulation towards them. The study also investigated the relationship between Machiavellianism and six friendship functions and found consistent results with five of the six functions, with women reporting lower scores on these five friendships functions. Future research should include both members of the friendship dyad in order to investigate the influence of Machiavellianism on emotional manipulation on both individuals.

\section{References}

Abell, L., Lyons, M., \& Brewer, G. (2014). The relationship between parental bonding, Machiavellianism and adult friendship quality. Individual Differences Research, 12, 191-197.

Abell, L., \& Brewer, G. (2014). Machiavellianism, self-monitoring, self-promotion and relational aggression on Facebook. Computers in Human Behavior, 36, 258-262.

Archer, J., \& Coyne, S. M. (2005). An integrated review of indirect, relational, and social aggression. Personality and Social Psychology Review, 9, 212-230.

Austin, E. J., Farrelly, D., Black, C., \& Moore, H. (2007). Emotional intelligence, Machiavellianism and emotional manipulation: Does EI have a dark side? Personality and Individual Differences, 43, 179-189.

Austin, E. J., \& O’Donnell, M. (2013). Development and preliminary validation of a scale to assess 
managing the emotions of others. Personality and Individual Differences, 55, 834-839.

Baron-Cohen, S., \& Wheelwright, S. (2003). The Friendship Questionnaire: An investigation of adults with Asperger Sydrome or high functioning Autism, and normal sex differences. Journal of Autism and Developmental Disorders, 33, 509-517.

Benenson, J. F., \& Christakos, A. (2003).The greater fragility of females' versus males' closest samesex friendships. Child Development, 74, 1123-1129.

Black, P. J., Woodworth, M., \& Porter, S. (2014). The Big Bad Wolf? The relation between the Dark Triad and the interpersonal assessment of vulnerability. Personality and Individual Differences, 67, 52-56.

Blieszer, R., \& Adams, R. G. (1992). Adult Friendship. Newbury Park, CA: Sage.

Booth-Kewley, S., Larson, G. E., \& Miyoshi, S. K. (2007). Social desirability effects on computerized and paper-and-pencil questionnaires. Computers in Human Behavior, 23, 463-477.

Campbell, A. (1999). Staying alive: Evolution, culture, and women's intrasexual aggression. Behavioral and Brain Sciences, 22, 203-252.

Christie, R., \& Geis, F. L. (1970). Studies in Machiavellianism. London: Academic Press.

David-Barrett T., Rotkirch A., Carney J., Behncke Izquierdo I., Krems J. A., et al. (2015). Women favour dyadic relationships, but men prefer clubs: Cross-cultural evidence from social networking. PLoS ONE, 10, e0118329.

Duffy P. J., Shiflett, S., \& Downey, R. G. (1977). Locus of control: Dimensionality and predictability using Likert scales. Journal of Applied Psychology, 62, 214-219.

Grovle, L., Haugen, A. J., Keller, A., Natvig, B., Brox, J. I., \& Grotle, M. (2012). Poor agreement found between self-report and a public registry on duration of sickness absence. Journal of Clinical Epidemiology, 65, 212-218.

Holden, R. R., Wheeler, S., \& Marjanovic, Z. (2012). When does random responding distort selfreport personality assessment? An example with the NEO PI-R. Personality and Individual Differences, 52, 15-20.

Jonason, P. K., \& Webster, G. D. (2012). A protean approach to social influence: Dark Triad personalities and social influence tactics. Personality and Individual Differences, 52, 521- 
526.

Jonason, P. K., \& Schmitt, D. P. (2012). What have you done for me lately? Friendship-selection in the shadow of the Dark Triad traits. Evolutionary Psychology, 10, 400-421.

Ináncsi, T., Láng, A. G., \& Bereczkei, (2015). Machiavellianism and adult attachment in general interpersonal and close relationships. Europe's Journal of Psychology, 11, 139-154.

Lyons, M., \& Aitken, S. (2010). Machiavellian friends? The role of Machiavellianism in friendship formation and maintenance. Journal of Social, Evolutionary and Cultural Psychology, 4, 194202.

Miller-Ott, A. E., \& Kelly, L. (2013). Mean girls in college: An analysis of how college women communicatively construct and account for relational aggression. Women's Studies in Communication, 36, 330-347.

Schmitt, M., Gollwitzer, M., Maes, J., \& Arbach, D. (2005). Justice Sensitivity: Assessment and Location in the Personality Space. European Journal of Psychological Assessment, 21, 202211.

Sherry, S. B., Hewitt, P. L., Besser, A., Flett, G. C., \& Klein, C. (2006). Machiavellianism, trait perfectionism, and perfectionistic self-presentation. Personality and Individual Differences, 40, 829-839.

Silverman, I., \& Choi, J. (2005). Locating places. In D. Buss (Ed.), The Handbook of Evolutionary Psychology (pp.177-199). Hoboken, NJ: John Wiley \& Sons.

Su, R., Rounds, J., \& Armstrong, P. I. (2009). Men and things, women and people: A meta-analysis of sex differences in interests. Psychological Bulletin, 135, 859-884.

Wai, M., \& Tiliopoulos, N. (2012). The affective and cognitive empathic nature of the dark triad of personality. Personality and Individual Differences, 52, 794.799.

Wastell, C., \& Booth, A. (2003). Machiavellianism: An Alexithymic Perspective. Journal of Social and Clinical Psychology, 22, 730-744.

Wilson, D. S., Near, D., \& Miller, R. R. (1996). Machiavellianism: A Synthesis of the Evolutionary and Psychological Literatures. Psychological Bulletin, 119, 285-299.

Veselka, L., Aitken Schermer, J., \& Vernon, P. A. (2011). Beyond the big five: The dark triad and the 
supernumerary personality inventory. Twin Research and Human Genetics, 14, 158-168.

Vigil, J. M. (2007). Asymmetries in the friendship preferences and social styles of men and women. Human Nature, 18, 143-161.

Xie, H., Cairns, B. D., \& Cairns, R. B. (2005). The development of aggressive behaviours among girls: Measurement issues, social functions, and differential trajectories. In D. J. Pepler, K. C. Masden, C. Webster \& K. S. Levenes (Eds.), The development and treatment of girlhood aggression (pp.105-136). Mahwah, NJ: Erlbaum. 
Table 1: Means and Correlations between Machiavellianism, Emotional Manipulation (Including Worsening and Inauthentic Strategies), and Friendship Functions

\begin{tabular}{|c|c|c|c|c|c|c|c|c|c|c|c|c|c|c|}
\hline & Mean & 1 & 2 & 3 & 4 & 5 & 6 & 7 & 8 & 9 & 10 & 11 & 12 & 13 \\
\hline 1 & 27.55 & & $.35 * *$ & $-.21 * *$ & $-.29 * *$ & $-.21 * *$ & $-.37 * *$ & $-.31 * *$ & $-.17 *$ & -.10 & -.02 & -.05 & -.02 & -.02 \\
\hline 2 & 123.58 & & & -.12 & -.08 & -.10 & $-.17 *$ & $-.22 * *$ & -.06 & -.07 & .02 & .14 & .07 & .06 \\
\hline 3 & 69.73 & & & & $.32 * *$ & $.28 * *$ & $.39 * *$ & $.37 * *$ & $-.27 * *$ & $-.32 * *$ & $-.26 * *$ & $-.35 * *$ & $-.73 * *$ & $-.27 * *$ \\
\hline 4 & 25.64 & & & & & $.67 * *$ & $.54 * *$ & $.54 * *$ & -.04 & $-.14 * *$ & $-.17 *$ & $-.15 *$ & -.16 & $-.20 * *$ \\
\hline 5 & 24.78 & & & & & & $.49 * *$ & $.51 * *$ & -.07 & -.13 & $-.19 * *$ & $-.18 * *$ & $-.19 * *$ & $-.18 *$ \\
\hline 6 & 13.20 & & & & & & & $.69 * *$ & $-.27 * *$ & $-.33 * *$ & $-.32 * *$ & $-.31 * *$ & $-.34 * *$ & $-.35 * *$ \\
\hline 7 & 19.89 & & & & & & & & $-.25 * *$ & $-.33 * *$ & $-.30 * *$ & $-.31 * *$ & $-.32 * *$ & $-.37 * *$ \\
\hline 8 & 34.54 & & & & & & & & & .71 & $.61^{* *}$ & $.54 * *$ & $.70 * *$ & $.66^{* *}$ \\
\hline 9 & 31.68 & & & & & & & & & & $.63 * *$ & $.59 * *$ & $.76 * *$ & $.76^{* *}$ \\
\hline 10 & 34.46 & & & & & & & & & & & $.61 * *$ & $.60 * *$ & $.68 * *$ \\
\hline 11 & 36.71 & & & & & & & & & & & & $.66 * *$ & $.62 * *$ \\
\hline 12 & 32.25 & & & & & & & & & & & & & $.76^{* *}$ \\
\hline 13 & 33.39 & & & & & & & & & & & & & \\
\hline
\end{tabular}

1 = Age, 2 = Friendship Length, $3=$ Machiavellianism, $4=$ Emotional Manipulation, $5=$ Emotional Manipulation Friend, $6=$ Worsen, $7=$ Inauthentic, $8=$ Companionship, $9=$ Help, $10=$ Intimacy, $11=$ Reliable alliance, $12=$ Self-Validation, $13=$ Emotional Security

Note*Correlation is significant at the 0.05 level $* *$ Correlations is significant at the 0.01 level 
Table 2: Means and Correlations between Machiavellianism, Emotional Manipulation Frequency, and Friendship Functions

\begin{tabular}{|c|c|c|c|c|c|c|c|c|c|c|c|c|}
\hline & Mean & 1 & 2 & 3 & 4 & 5 & 6 & 7 & 8 & 9 & 10 & 11 \\
\hline 1 & 23.65 & & $.37 * *$ & -.10 & $-.31 * *$ & $-.28 * *$ & .01 & -.02 & $.20 * *$ & .10 & .10 & $.16^{* *}$ \\
\hline 2 & 112.59 & & & -.11 & $-.21 * *$ & $-.24 * *$ & .07 & .05 & .14 & $.31 * *$ & $.20 * *$ & $.17 *$ \\
\hline 3 & 68.82 & & & & $.35^{* *}$ & $.27 * *$ & -.14 & -.14 & $-.19 * *$ & $-.17 *$ & -.12 & $-.22 * *$ \\
\hline 4 & 15.01 & & & & & $.74 * *$ & $-.16^{*}$ & $-.15^{*}$ & $-.24 * *$ & $-.24 * *$ & -.13 & $-.21 * *$ \\
\hline 5 & 15.95 & & & & & & $-.21 * *$ & $-.24 * *$ & $-.26 * *$ & $-.35^{* *}$ & $-.30 * *$ & $-.38 * *$ \\
\hline 6 & 34.06 & & & & & & & $.64 * *$ & $.64 * *$ & $.47 * *$ & $.68 * *$ & $.64 * *$ \\
\hline 7 & 32.47 & & & & & & & & $.66^{* *}$ & $.54 * *$ & $.67 * *$ & $.68 * *$ \\
\hline 8 & 34.54 & & & & & & & & & $.62 * *$ & $.62 * *$ & $.69 * *$ \\
\hline 9 & 35.53 & & & & & & & & & & $.53 * *$ & $.59 * *$ \\
\hline 10 & 32.63 & & & & & & & & & & & $.76^{* *}$ \\
\hline 11 & 32.86 & & & & & & & & & & & \\
\hline
\end{tabular}

1 = Age, 2 = Friendship Length, 3 = Machiavellianism, $4=$ Emotional Manipulation Frequency, $5=$ Emotional Manipulation Friend Frequency, $6=$ Companionship, $7=$ Help, $8=$ Intimacy, $9=$ Reliable alliance, $10=$ Self-Validation, $11=$ Emotional Security

Note $*$ Correlation is significant at the 0.05 level $* *$ Correlations is significant at the 0.01 level 